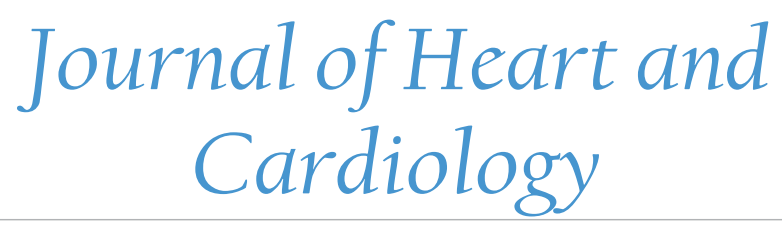

\title{
Electrocardiogram Characteristics that Distinguish Perimitral Reentry from Other Atrial Tachycardias after Atrial Fibrillation Ablation
}

\author{
Hironori Sato $^{1,2^{*}}$, Yasuteru Yamauchi ${ }^{3}$, Koji Azegami ${ }^{1}$, Takamichi Miyamoto ${ }^{4}$, Kenichiro Otomo ${ }^{5}$, Ken- \\ zo Hirao $^{6}$, Mitsuaki Isobe ${ }^{2}$
}

${ }^{1}$ Department of Cardiovascular Medicine, Shinyurigaoka General Hospital, Kawasaki, Japan

${ }^{2}$ Department of Cardiovascular Medicine, Tokyo Medical and Dental University, Tokyo, Japan

${ }^{3}$ Heart Center, Yokohama-City Bay Red Cross Hospital, Yokohama, Japan

${ }^{4}$ Department of Cardiology, Musashino Red Cross Hospital, Tokyo, Japan

${ }^{5}$ Department of Cardiovascular Medicine, Ome Municipal General Hospital, Tokyo, Japan

${ }^{6}$ Heart Rhythm Center, Tokyo Medical and Dental University, Tokyo, Japan

*Corresponding author: Hironori Sato, MD, Department of Cardiovascular Medicine, Tokyo Medical and Dental University, Yushima 1-5-45, Bunkyoku, Tokyo 113-8519, Japan, Tel: + 8135803 5951; Fax: + 81358030238 ;

E-mail: satohironorihr@gmail.com

\begin{abstract}
Introduction: Ablation of Atrial Fibrillation (AF) can induce several types of Atrial Tachycardias (ATs). Perimitral reentry is a common mechanism among postablation ATs. The electrocardiographic (ECG) criteria for differentiating perimitral reentry from other postablation ATs may be useful in clinical practice.

Methods and results: A total of 48 consecutive post atrial fibrillation ablation patients with AT which occurred during ablation of recurrent atrial fibrillation were enrolled. We analyzed surface 12-lead ECG of 61 ATs. As for the patients, their doctors decided whether to stop antiarrhythmic drugs before ablation. We analyzed the ECG of the first 30 ATs in 24 patients, and developed an algorithm for differentiating perimitral reentry from other ATs based on P-wave morphology. We then prospectively evaluated this algorithm in the following 31 ATs in 24 patients. The polarity of $\mathrm{P}$ waves during AT were classified as positive, negative, positive/negative, negative/positive, or isoelectric in each of the 12 leads. The mechanism of 30 ATs were identified as perimitral reentry $(n=15)$, other macroreentry $(n=7)$, centrifugal AT $(n=5)$, gap-related left atrial and pulmonary vein reentry $(n=2)$, and tachycardia within reconnected PV $(n=1)$. $\mathrm{P}$ waves in perimitral reentry displayed three morphological features: 1) a positive $\mathrm{P}$ wave in lead V1 (sensitivity 87\%), 2) isoelectric P wave in at least one precordial lead (sensitivity 93\%), and 3) no negative or biphasic $\mathrm{P}$ wave in precordial leads (sensitivity $93 \%$ ). In the prospective evaluation, an algorithm based on the above three criteria resulted in an overall accuracy of $81 \%$ in predicting whether AT was due to perimitral reentry or not.
\end{abstract}

Conclusions: We report a new P-wave algorithm using only the precordial leads that helps distinguish perimitral reentry from other postablation ATs.
Received Date: October 28, 2016

Accepted Date: December 24, 2016

Published Date: January 03, 2017

Citation: Sato, H., et al. Electrocardiogram Characteristics that Distinguish Perimitral Reentry from Other Atrial Tachycardias after Atrial Fibrillation Ablation. (2017) J Heart Cardiol 3(1): 1- 6.

DOI: $10.15436 / 2378-6914.17 .1192$

Keywords: Pulmonary vein isolation, Precordial leads, P-wave morphology, Algorithm

\section{Introduction}

Catheter ablation is recognized as an effective treatment for Atrial Fibrillation (AF) ${ }^{[1]}$. According to AHA/ACC/ HRS Guideline, catheter ablation for symptomatic paroxysmal AF refractory or intolerant to antiarrhythmic medication is cat- egorized as Class I, and persistent AF refractory or intolerant to antiarrhythmic medication is categorized as Class IIa. It is expected that the maintenance of sinus rhythm by catheter ablation with heart failure and AF improves cardiac function ${ }^{[2]}$. High-burden paroxysmal/persistent AF patients can obtain higher freedom from AF by performing AF ablation ${ }^{[3]}$. Thus, AF ab- 
lation is extremely useful to reduce AF burden, the progression of chronic arrhythmic disease, and heart failure. Maintenance of sinus rhythm after ablation is associated with a reduction in cardiovascular mortality in patients with $\mathrm{AF}^{[4,5]}$. Pulmonary Vein Isolation (PVI) is widely accepted as the cornerstone of ablation strategy. However, AF ablation, especially extensive left atrial ablation procedures in addition to PVI, can give rise to new-onset organized Atrial Tachycardias (AT ${ }^{[6-10]}$. Mechanisms of these arrhythmias are usually diagnosed by entrainment maneuvers, Post-Pacing Interval (PPI) analysis, and the use of 3-dimensional mapping systems. The most common mechanism of postablation AT is perimitral reentry ${ }^{[10-12]}$. AT that occur after AF ablation can cause even more severe symptoms than those from original arrhythmia prior to the index ablation procedure, since they are often incessant and associated with rapid ventricular response. Moreover, recurrent atrial arrhythmia is difficult to control using antiarrhythmic medications and is not always found at the time of consultation. Continuous monitoring is very useful for detecting recurrent $\mathrm{AF}$ and new-onset $\mathrm{AT}^{[13]}$.

Twelve-lead ECGs play a key role in understanding arrhythmias and are useful for identifying the mechanism and origin of tachycardias because they record the electrical activity of the whole heart. However, ways to distinguish perimitral reentry from other postablation ATs using 12-lead ECGs have not been established. The aims of this study were, 1) to analyze P-wave morphology of postablation ATs and identify ECG characteristics of perimitral reentry, and 2) to develop an algorithm using that information to distinguish perimitral reentry from other ATs. We used the first half of our patients to develop the algorithm, and prospectively evaluated the validity of this algorithm in the remaining half of the patients.

\section{Methods}

\section{Study population}

This study included a consecutive series of 61 sustained ATs occurring in 48 patients during the Electrophysiological (EP) test and ablation for the recurrence of atrial arrhythmia after prior circumferential antral PVI at two hospitals (Musashino Red Cross Hospital (Tokyo, Japan) and Ome Municipal General Hospital (Tokyo, Japan)) between June 2011 and March 2014. The enrolled ATs were ATs that were terminated by catheter ablation and ATs which cycle length prolonged by catheter ablation if the mechanism was macroreentry. Patients with a past history of open heart surgery were excluded.

We retrospectively analyzed the $\mathrm{P}$ wave of 12-lead ECGs of the first 24 patients, corresponding to 30 ATs. The diagnostic ECG algorithm for identifying perimitral reentry was constructed according to the results of that analysis. The algorithm was then prospectively tested in the remaining 24 consecutive patients with 31 ATs by a blinded observer. All patients provided written informed consent prior to the procedure. The study was approved by the Institutional Review Boards at the respective hospitals.

\section{ECG analysis}

The polarity of the $\mathrm{P}$ waves was classified into 5 types $^{[14]}$ : 1) positive $=\mathrm{P}$ waves with deflections above the isoelectric line (the T-P segment);

2) negative $=P$ waves with deflections below the isoelectric line;
3) positive/negative $=\mathrm{P}$ waves having a positive and then negative deflection;

4) negative/positive $=P$ waves having a negative and then positive deflection;

5) isoelectric $=\mathrm{P}$ waves having an absolute amplitude of $\leq 0.05$ $\mathrm{mV}$.

Interpretations were performed using standard ECG recordings. We did not use magnification. The P-wave morphology was analyzed at areas without superimposed $\mathrm{T}$ waves.

\section{Classification of ATs}

Four types of AT can develop after AF ablation based on mechanism, macroreentry, centrifugal AT, PV tachycardia with conduction gap and LA-PV reentrant tachycardia ${ }^{[15]}$. Macroreentry was defined as reentry around a large central obstacle. Entrainment maneuvers were performed to confirm the diagnosis. In cases of post pacing interval exceeding the Cycle Length (CL) by no more than $30 \mathrm{~ms}$ in 2 separate sites, the only possibility was macroreentry. Centrifugal AT was defined as atrial activity originating from a single focus and spreading centrifugally according to the activation map and conventional measurements. If activity accounting for $>75 \%$ of the AT CL was present in an area with a diameter $<3 \mathrm{~cm}$, localized reentry was considered ${ }^{[16]}$. Localized reentry was included in centrifugal AT. PV tachycardia with conduction gap originated from the muscle sleeve of the reconnected PV. The circuit of LA-PV reentrant tachycardia included the myocardium within the PV and LA via 2 conduction gaps arising in a previous circumferential lesion. Entrainment mapping showed that the LA and PVs were involved in the reentrant circuit.

\section{Mapping and ablation of ATs}

The ablation was performed under sedation with dexmedetomidine. Surface ECGs and endocardial electrograms were continuously monitored and stored on a computer-based digital amplifier/recorder system (Cardio Lab System, GE Healthcare, Milwaukee, WI, or CARDIO MASTER, Nihon Kohden, Tokyo, Japan) during the EP study and ablation. The 12-lead ECG was recorded at a $25 \mathrm{~mm} / \mathrm{s}$ sweep speed with filter settings of $0.05 \mathrm{~Hz}$ (high pass) and $100 \mathrm{~Hz}$ (low pass).

When persistent AT occurred during EP test or ablation of AF, we investigated the origin and the mechanism of AT using conventional entrainment techniques and an electro-anatomic mapping system (CARTO, Biosense-Webster) for activation mapping. The ablation catheter was a $3.5-\mathrm{mm}$ externally irrigated tip ablation catheter (NAVISTAR Thermo-Cool, Biosense-Webster). Radiofrequency ablation was performed at a power of $30 \mathrm{~W}$ (maximum, $35 \mathrm{~W}$; posterior left atrial wall, 25 $\mathrm{W}$ ), a maximum flow rate of $30 \mathrm{~mL} / \mathrm{min}$, and a maximum temperature of $42^{\circ} \mathrm{C}$. For macroreentry, the linear ablation lesions were established by crossing the critical isthmus. For centrifugal ATs, the radiofrequency energy was applied at the sites with the earliest activation. For PV tachycardia and LA-PV reentrant tachycardia, the ablation sites were conduction gaps in the previous circumferential lesion between the LA and PV.

\section{Prospective evaluation of the algorithm}

An algorithm was developed based on analysis of the P wave morphology in the 30 ATs from the first 24 patients. The algorithm was then prospectively applied to 31 ATs from the re- 
maining 24 patients by a blinded observer. This observer differed from the cardiologist who developed the algorithm.

\section{Statistical analysis}

Continuous variables were presented as mean \pm standard deviation. Fisher's exact test was used for evaluating the significance of the differences between categorical variables. A P value of $<$ 0.05 was considered statistically significant.

\section{Results}

\section{Patient characteristics}

Electrocardiograms of 30 ATs in 24 consecutive patients who underwent AT ablation at our institutions were reviewed. There were 8 ATs that occurred during use of antiarrhythmic drug and 22 ATs that were not using antiarrhythmic drug. The mean age was $64 \pm 10$ years. Three patients had mild mitral stenosis and 1 patient had hypertrophic non-obstructive cardiomyopathy. The remaining patients had no structural heart disease. There were 5 patients with a prior history of stroke. No patients had cerebral ischemic events during ablation. The mean LA diameter was $44 \pm 7 \mathrm{~mm}$ in the parasternal window. Mean left ventricular ejection fraction was $67 \pm 9 \%$. All patients had undergone previous PVI. Before AT occurred, the right atrial isthmus was ablated in 22 ATs, LA roof line in 8 , and mitral isthmus line in 7. A further 8 received LA defragmentation ablation (Table 1). At our two hospitals, we routinely perform a PVI procedure in combination with a Cavotricuspid Isthmus (CTI) ablation in order to avoid a repeat to deal with masked CTI-dependent flutter. All patients who had received their initial AF treatment at our two hospitals had undergone PVI and CTI ablation, but some patients who had received initial AF treatment at different hospitals had not undergone CTI ablation.

Table 1: Clinical Characteristics of the 24 Patients with 30 ATs who's Data was used for Determination of Perimitral Reentry ECG Characteristics.

\begin{tabular}{|l|c|}
\hline \multicolumn{2}{|c|}{ Demographic characteristics } \\
\hline Median age (years) & $63.8 \pm 9.5$ \\
\hline Sex & 21 male, 9 female \\
\hline Clinical characteristics \\
\hline Heart failure, no, (\%) & $5(17 \%)$ \\
\hline Hypertension, no, (\%) & $9(30 \%)$ \\
\hline Diabetes mellitus, no, (\%) & $2(7 \%)$ \\
\hline Stroke, no, (\%) & $5(17 \%)$ \\
\hline CHADS2 score & $1 \pm 1.3$ \\
\hline Structural heart disease, no, (\%) & $5(17 \%)$ \\
\hline Echocardiographic measures & $43.7 \pm 6.7$ \\
\hline Left atrial size, mm & $67.1 \pm 9.0$ \\
\hline Ejection fraction, $\%$ & \\
\hline Procedure & $30(100 \%)$ \\
\hline PV isolation, no, (\%) & $22(73 \%)$ \\
\hline Cavotricuspid isthmus line, no, (\%) & $8(27 \%)$ \\
\hline LA Roof line, no, (\%) & $7(23 \%)$ \\
\hline LA Mitral line, no, (\%) & $8(27 \%)$ \\
\hline LA defragmentation, no, (\%) & \\
\hline
\end{tabular}

\section{Mechanism of AT}

The mechanisms responsible for AT were macroreentry in 21 ATs, figure-of- 8 with a perimitral and roof-dependent loop in 1 AT, centrifugal AT in 5 ATs, tachycardia within the reconnected PV in 1 AT (left superior PV, $\mathrm{n}=1$ ), and LA-PV reentrant tachycardia in 2 ATs (left superior PV, $\mathrm{n}=1$, right superior $\mathrm{PV}, \mathrm{n}$ $=1$ ). In the figure-of- 8 reentry, we first performed mitral isthmus line ablation, causing the AT to change into a solely roof-dependent loop. That AT was terminated during the ablation of the LA roof line. Centrifugal ATs were found to originate in the LA appendage $(\mathrm{n}=2)$, LA anterior wall $(\mathrm{n}=1)$, RA septum $(\mathrm{n}=$ $1)$, and the crista terminalis $(\mathrm{n}=1)$. The macroreentrant circuit was diagnosed as perimitral reentry in 15 ATs ( 8 were clockwise and 7 were counter clockwise), roof dependent AT in 5 ATs, and CTI-dependent reentry in 1 AT. Only one patient showed both clockwise and counterclockwise perimitral reentry during the same session.

The cycle length of AT was $354 \pm 98 \mathrm{~ms}$ in centrifugal, $370 \mathrm{~ms}$ in PV tachycardia, $290 \pm 85 \mathrm{~ms}$ in LA-PV reentrant tachycardia, $303 \pm 92 \mathrm{~ms}$ in macroreentry, and $330 \mathrm{~ms}$ in figure-of- 8 reentry.

\section{Characteristics of P-wave morphology}

The P-wave morphologies for perimitral reentry and other ATs are presented in Table 2. Examples of ECG characteristics typical of AT from different mechanisms are shown in Figure 1.

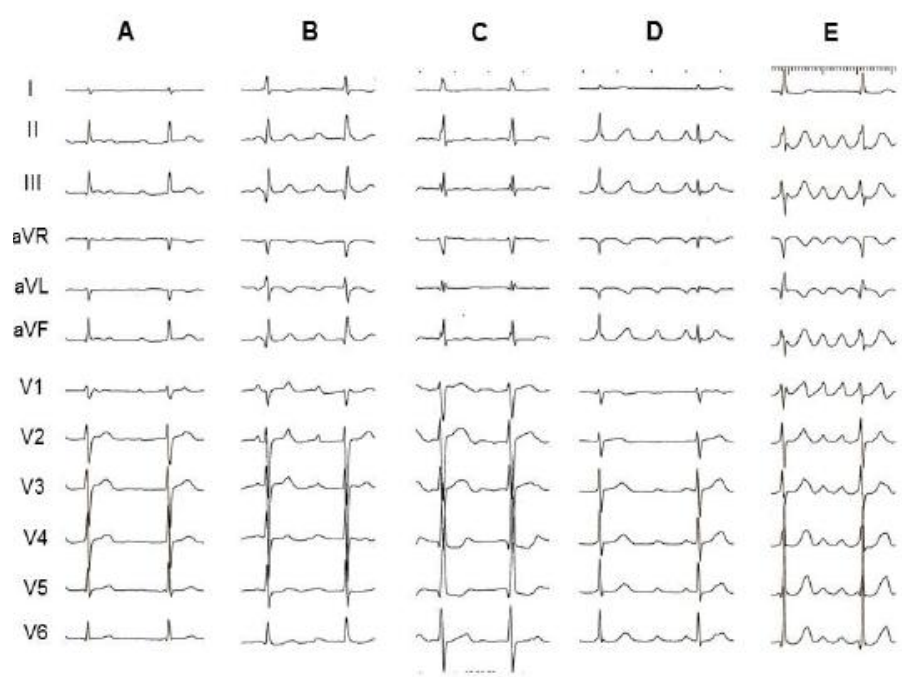

Figure 1: Representative ECG examples of different mechanism of AT. A: perimitral reentry. The $\mathrm{P}$ wave in lead $\mathrm{V} 1$ is positive. Isoelectric $\mathrm{P}$ waves are seen between V3 and V6. In lead II, III and aVF, P waves show a positive deflection. B: roof-dependent reentry. In this case, all precordial leads display positive $P$ waves. C: centrifugal AT originating from the anterior LA. This case had negative $\mathrm{P}$ waves in the precordial leads. D: electrical reconnection between LA and LSPV. This case did not have a positive $\mathrm{P}$ wave in lead V1. E: gap related LA and LSPV reentry. In this case, all precordial leads display positive $\mathrm{P}$ waves. 
ECG characteristics of perimitral reentry after AF ablation

Table 2: Atrial Tachycardia P-wave Morphology in the 12 Lead ECG.

\begin{tabular}{|c|c|c|c|c|c|c|c|c|c|c|c|}
\hline \multirow{2}{*}{ Lead } & \multicolumn{5}{|c|}{ Perimitral reentry $(n=15)$} & \multicolumn{6}{|c|}{ Non- perimitral reentry $(n=15)$} \\
\hline & positive & negative & $+/-$ & $-/+$ & iso & positive & negative & $+/-$ & $-/+$ & iso & P Value \\
\hline I & 3 & 0 & 0 & 0 & 12 & 3 & 3 & 0 & 0 & 9 & 0.352 \\
\hline II & 14 & 0 & 0 & 1 & 0 & 9 & 2 & 2 & 1 & 1 & 0.177 \\
\hline III & 14 & 0 & 0 & 1 & 0 & 9 & 2 & 2 & 1 & 1 & 0.177 \\
\hline aVR & 0 & 11 & 1 & 0 & 3 & 3 & 8 & 1 & 0 & 3 & 0.381 \\
\hline aVL & 0 & 10 & 0 & 0 & 5 & 2 & 9 & 0 & 2 & 2 & 0.176 \\
\hline aVF & 14 & 1 & 0 & 0 & 0 & 10 & 2 & 2 & 1 & 0 & 0.3 \\
\hline $\mathrm{V} 1$ & 13 & 0 & 1 & 0 & 1 & 10 & 1 & 3 & 0 & 1 & 0.651 \\
\hline $\mathrm{V} 2$ & 7 & 0 & 1 & 0 & 7 & 9 & 2 & 1 & 1 & 2 & 0.144 \\
\hline V3 & 5 & 0 & 0 & 0 & 10 & 8 & 2 & 1 & 1 & 3 & 0.031 \\
\hline V4 & 6 & 0 & 0 & 0 & 9 & 7 & 3 & 0 & 0 & 5 & 0.145 \\
\hline V5 & 4 & 0 & 0 & 0 & 11 & 7 & 3 & 0 & 2 & 3 & 0.009 \\
\hline V6 & 4 & 0 & 0 & 0 & 11 & 7 & 2 & 0 & 2 & 4 & 0.039 \\
\hline
\end{tabular}

counterclockwise

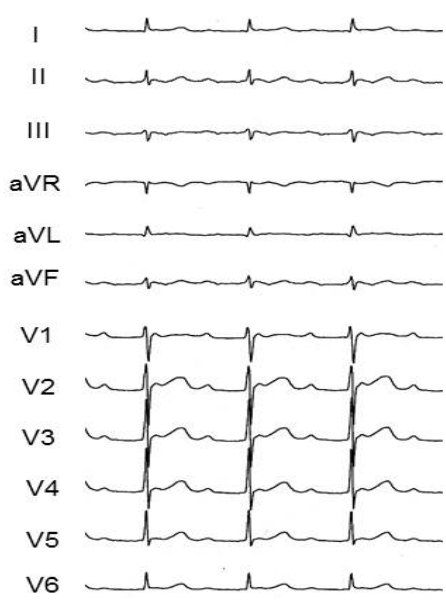

clockwise

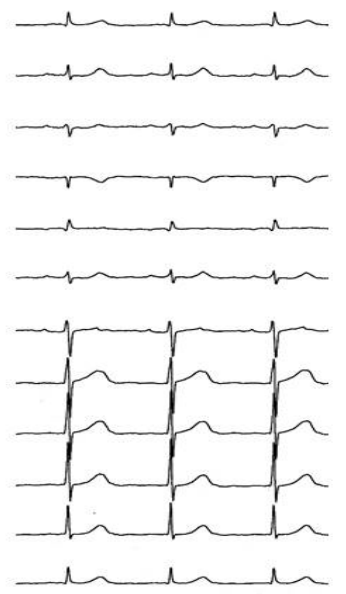

In the limb leads, 14 (93\%) perimitral reentries showed positive $\mathrm{P}$ wave polarity in leads II, III and aVF. The deflection in leads II, III and aVF in the patient who showed both directions of perimitral reentry in the same session did not change (Figure 2). Most perimitral reentry showed isoelectric and negative $\mathrm{P}$ waves in leads I and aVL, respectively. However, the other postablation ATs had deflection of $\mathrm{P}$ wave in the limb leads similar to perimitral reentry.

In the precordial leads, $13(87 \%)$ perimitral reentries had a positive $\mathrm{P}$ wave in lead V1. Isoelectric $\mathrm{P}$ wave in at least 1 precordial lead was shown in $14(93 \%)$ perimitral reentries and 7 $(50 \%)$ other postablation ATs $(\mathrm{P}=0.014)$. No perimitral reentry had a negative $\mathrm{P}$ wave and $1(7 \%)$ had a biphasic $\mathrm{P}$ wave in the precordial leads (Table 3). Among perimitral reentry, $2(13 \%)$ occurred before CTI ablation. One had positive polarity in V1 and V2, and isoelectric P waves in between V3 and V6. The other showed isoelectric P waves between V1 and V6.

Figure 2: Surface ECG of both counterclockwise and clockwise perimitral reentries occurring in the same patient during the same session. Both tachycardias have positive $\mathrm{P}$ waves in leads II, III and aVF.

Table 3: Performance of Predictors of Perimitral Reentry.

\begin{tabular}{|c|c|c|c|c|c|c|c|c|}
\hline Criteria & TP & FP & FN & TN & Se (\%) & Sp (\%) & PPV (\%) & NPV (\%) \\
\hline 1) Positive $P$ wave in lead V1 & 13 & 10 & 2 & 5 & 87 & 33 & 57 & 71 \\
\hline $\begin{array}{l}\text { 2) Isoelectric } P \text { wave in at least } 1 \\
\text { precordial lead }\end{array}$ & 14 & 7 & 1 & 8 & 93 & 53 & 67 & 89 \\
\hline $\begin{array}{l}\text { 3) No negative or biphasic } P \text { wave } \\
\text { in precordial leads }\end{array}$ & 14 & 7 & 1 & 8 & 93 & 53 & 67 & 89 \\
\hline 4) 1$)+2)+3)$ & 13 & 1 & 2 & 14 & 87 & 93 & 93 & 88 \\
\hline
\end{tabular}

FN = False Negatives; FP = False Positives; NPV = Negative Predictive Value; Se = Sensitivity; $\mathrm{Sp}=$ Specificity; $\mathrm{TN}=\mathrm{True}$ Negatives; $\mathrm{TP}=$ True Positives.

\section{Development of the algorithm and prospective examination}

Figure 3 demonstrates our new diagnostic algorithm for differentiating perimitral reentry from other postablation ATs. This algorithm relies only on precordial leads. Perimitral reentry was diagnosed if all 3 features which were observed in the retrospective study were present. In this algorithm, among 30 ATs which were retrospectively reviewed, sensitivity, specificity, the Positive Predictive Value (PPV), and Negative Predictive Values
(NPV) were $87 \%, 93 \%, 93 \%$, and $88 \%$, respectively, for identifying perimitral reentry (Table 3 ). 


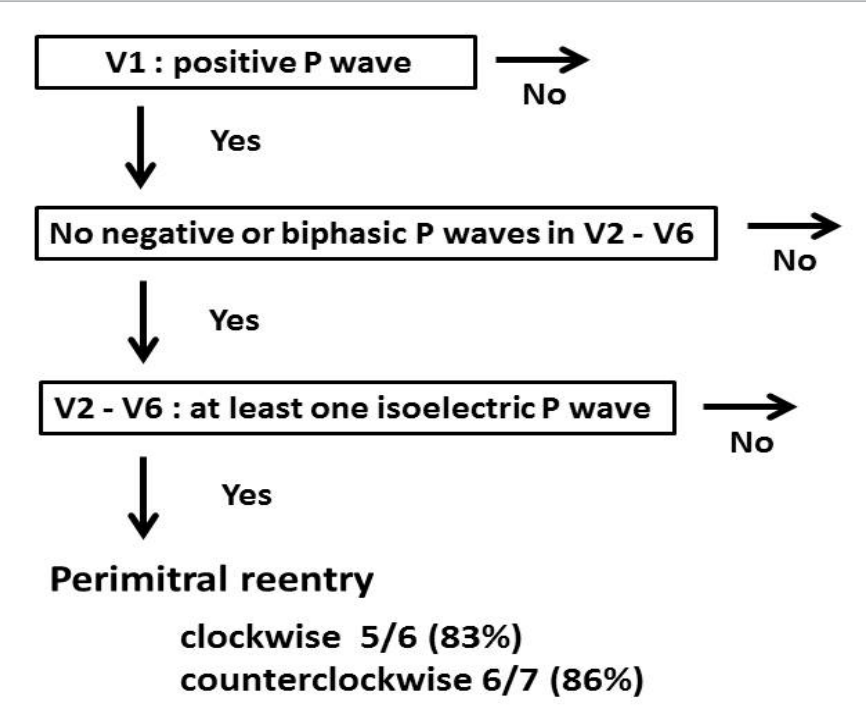

Figure 3: A new diagnostic algorithm for differentiating perimitral reentry based on the surface electrocardiogram. In the prospective part of the study, there were 13 patients with perimitral reentry, 6 with clockwise, 7 with counter-clockwise. ECG characteristics were able to identify rotation direction in 5 of 6 clockwise and in 6 of 7 counter-clockwise perimitral reentries.

Subsequently, the last 31 ATs were examined to validate the algorithm for diagnosing the perimitral reentry. These ATs included 13 perimitral reentries (6 were clockwise and 7 were counter clockwise), 2 roof dependent ATs, 3 cavotricuspid isthmus-dependent reentries, 1 figure-of- 8 with a perimitral and roof-dependent loop, 1 tachycardia within reconnected RSPV, 1 LA-PV reentrant tachycardia, and 10 centrifugal AT ( 6 originated from the LA anterior wall, 1, the LA posterior wall, and 3, the LA septal wall). A blind experiment was conducted with a different cardiologist who did not know the mechanism of ATs. The blinded cardiologist determined whether AT mechanism was perimitral reentry or not using the algorithm. The algorithm's sensitivity, specificity, PPV, and NPV were $85 \%, 78 \%, 73 \%$, and $88 \%$, respectively. The ATs found to be false positive were centrifugal ATs, especially from the LA anterior wall. The algorithm was unable to diagnose perimitral reentry in cases where the perimitral reentry did not exhibit positive $\mathrm{P}$ waves in the V1 lead.

\section{Discussion}

\section{Main findings}

We studied the morphology of P waves of AT arising after PV isolation. Perimitral reentry and other postablation ATs displayed distinguishing characteristics in the precordial leads, but were similar in the limb leads in this study. Most postablation ATs showed positive deflection in the inferior limb leads. Perimitral reentry also had positive $P$ waves in the inferior leads regardless of the direction of rotation. Perimitral reentry showed positive deflections in lead V1. Between V2 and V6, perimitral reentry had at least one isoelectric $\mathrm{P}$ wave and did not show any negative or biphasic $\mathrm{P}$ wave. The algorithm that we created using the $\mathrm{P}$ wave morphology of the precordial leads was able to distinguish perimitral reentry from other postablation ATs in a prospective validation test.

\section{ECG characteristics of perimitral reentry}

Previous studies have also reported that perimitral re- entry has a positive $\mathrm{P}$ wave in $\mathrm{V} 1^{[17-20]}$. However, this finding was also true for other $\mathrm{ATs}^{[17]}$. Chang et al ${ }^{[18]}$ noted that a negative polarity in the precordial leads could differentiate RA from LA macroreentrant ATs. This is consistent with our data. The electrical activity vector of perimitral reentry is expected to be roughly perpendicular to the precordial leads. The electrical activity that moves toward and away from the precordial leads (especially V2-V6) is low in amplitude in perimitral reentry compared to that of other ATs. Therefore, perimitral reentry may have at least one isoelectric $\mathrm{P}$ wave in leads V2-V6 rather than clear positive or negative polarity. No previous studies have described this finding. The reason that the lead with isoelectric $\mathrm{P}$ wave is not fixed is probably because the anatomy and position of the left atrium varies among patients.

Gerstenfeld et $a{ }^{[19]}$ reported that the $\mathrm{F}$ wave vector was negative in leads I and aVL and positive in the inferior leads for counterclockwise perimitral reentry, and the converse were true for clockwise perimitral reentry. The latter observation could not be reproduced in the present study. Even in the patient having both clockwise and counterclockwise perimitral reentry in the same session, the polarity of $\mathrm{P}$ waves in the inferior leads did not change. The study populations of both their study and the present study were small. Whether the polarity of the limb leads can be reversed in clockwise perimitral reentry needs further investigation in a larger study.

\section{Cases difficult to diagnose by the new algorithm}

Our algorithm to identify perimitral reentry showed satisfactory results in the prospective validation part of the study. However, a few centrifugal ATs from the LA anterior wall were incorrectly diagnosed as perimitral reentry by the algorithm. Because the ratio of centrifugal AT from the LA anterior wall was higher in the prospective study than in the retrospective analysis, the specificity and the positive predictive value of the algorithm in the prospective study were reduced.

Perimitral reentry infrequently showed isoelectric or biphasic $\mathrm{P}$ wave in lead V1. When patients have received extensive LA ablation or have widespread scar area in the LA, perimitral reentry may not have a positive deflection in $\mathrm{V} 1$.

\section{Influence of atrial fibrosis}

Delayed enhancement Magnetic Resonance Imaging (MRI) is considered an effective modality to identify atrial fibrosis. It is reported that extensive fibrosis of the left atrial wall is associated with recurrent arrhythmia following catheter ablation $^{[21]}$. MRI is also capable of detecting ablation induced scarring $^{[22]}$. However, patients in our study did not undergo MRI since this examination is not performed in the routine care of AF patients. Recently, it was also reported that microRNA (miRs) was an effective modality to identify atrial fibrosis ${ }^{[23,24]}$. Plasma levels of miRs-21 and 150 were lower in patients with AF than in those without AF, and its levels increased after catheter ablation. This shows us that catheter ablation may induce reverse remodeling of the atrium.

\section{Clinical implication}

Some patients showed sustained AT at the outpatient clinic and returned to sinus rhythm before the EP test. The same AT as that seen in the outpatient clinic does not always occur during the EP test. If perimitral reentry can be diagnosed based on 12-lead ECGs in the outpatient clinic, mitral isthmus ablation 
might be considered even in cases where perimitral reentry was not induced during the EP test. Our new algorithm is useful for diagnosing perimitral reentry even when AT is recorded only in the 12-lead ECG.

\section{Study limitations}

Our study has several limitations that should be noted. The number of ATs in this study was small. The small sample size limits the ability to draw strong and generalizable conclusions. This study did not determine discontinuation criteria of antiarrhythmic drugs in electrophysiology test and ablation. We could not investigate the influence of antiarrhythmic drug on ECG and autonomic function ${ }^{[25]}$. Finally, most patients in this study received right atrial isthmus ablation during the first PVI session for the prevention of cavotricuspid isthmus-dependent reentry. Changing the electrical activation in the right atrium may influence the surface ECG morphology of ATs.

\section{Conclusion}

Isoelectric $\mathrm{P}$ wave in any lead between V2 and V6 suggests perimitral reentry among ATs occurring after AF ablation. We present a surface ECG based algorithm that uses only the precordial leads and differentiates perimitral reentry from other postablation ATs. Perimitral reentry can be identified simply by this algorithm and may facilitate planning of ablation strategy.

\section{References}

1. January, C.T., Wann, L.S., Alpert, J.S., et al. 2014 AHA/ACC/HRS guideline for the management of patients with atrial fibrillation: a report of the American College of Cardiology/American Heart Association Task Force on Practice Guidelines and the Heart Rhythm Society. (2014) J Am Coll Cardiol 64(21): e1-e76.

2. Hsu, L.F., Jais, P., Sanders, P., et al. Catheter ablation for atrial fibrillation in congestive heart failure. (2004) N Engl J Med 351: 2373-2383.

3. Verma, A., Mantovan, R., Macle, L., et al. Substrate and trigger ablation for reduction of atrial fibrillation (STAR AF): a randomized, multicentre, international trial. (2010) Eur Heart J 31(11): 1344-1356.

4. Marfella, R., Sasso, F.C., Siniscalchi, M., et al. Brief episodes of silent atrial fibrillation predict clinical vascular brain disease in type 2 diabetic patients. (2013) J Am Coll Cardiol 62(6): 525-530.

5. Ghanbari, H., Başer, K., Jongnarangsin, K., et al. Mortality and cerebrovascular events after radiofrequency catheter ablation of atrial fibrillation. (2014) Heart Rhythm 11(9): 1503-1511.

6. Sawhney, N., Anousheh, R., Chen, W., et al. Circumferential pulmonary vein ablation with additional linear ablation results in an increased incidence of left atrial flutter compared with segmental pulmonary vein isolation as an initial approach to ablation of paroxysmal atrial fibrillation. (2010) Circ Arrhythm Electrophysiol 3(3): 243-248.

7. Rostock, T., Drewitz, I., Steven, D., et al. Characterization, mapping, and catheter ablation of recurrent atrial tachycardias after stepwise ablation of long-lasting persistent atrial fibrillation. (2010) Circ Arrhythm Electrophysiol 3(2): 160-169.

8. Deisenhofer, I., Estner, H., Zrenner, B., et al. Left atrial tachycardia after circumferential pulmonary vein ablation for atrial fibrillation: incidence, electrophysiological characteristics, and results of radiofrequency ablation. (2006) Europace 8(8): 573-582.
9. Mesas, C.E., Pappone, C., Lang, C.C., et al. Left atrial tachycardia after circumferential pulmonary vein ablation for atrial fibrillation: electroanatomic characterization and treatment. (2004) J Am Coll Cardiol 44(5): 1071-1079.

10. Chae, S., Oral, H., Good, E., et al. Atrial tachycardia after circumferential pulmonary vein ablation of atrial fibrillation: mechanistic insights, results of catheter ablation, and risk factors for recurrence. (2007) J Am Coll Cardiol 50(18): 1781-1787.

11. Chang, S.L., Lin, Y.J., Tai, C.T., et al. Induced atrial tachycardia after circumferential pulmonary vein isolation of paroxysmal atrial fibrillation: electrophysiological characteristics and impact of catheter ablation on the follow-up results. (2009) J Cardiovasc Electrophysiol 20(4): 388-394.

12. Chugh, A., Oral, H., Lemola, K., et al. Prevalence, mechanisms, and clinical significance of macroreentrant atrial tachycardia during and following left atrial ablation for atrial fibrillation. (2005) Heart Rhythm 2(5): 464-471.

13. Sardu, C., Santamaria, M., Rizzo, M.R., et al. Telemonitoring in heart failure patients treated by cardiac resynchronisation therapy with defibrillator (CRT-D): the TELECART Study. (2016) Int J Clin Pract 70(7): 569-576.

14. Kistler, P.M., Roberts-Thomson, K.C., Haqqani, H.M., et al. P-wave morphology in focal atrial tachycardia: Development of an algorithm to predict the anatomic site of origin. (2006) J Am Coll Cardiol 48(5): 1010-1017.

15. Satomi, K. Electrophysiological characteristics of atrial tachycardia after pulmonary vein isolation of atrial fibrillation. (2010) Circ J 74(6): 1051-1058.

16. Jaïs, P., Matsuo, S., Knecht, S., et al. A deductive mapping strategy for atrial tachycardia following atrial fibrillation ablation: importance of localized reentry. (2009) J Cardiovasc Electrophysiol 20(5): 480-491.

17. Wasmer, K., Monning, G., Bittner, A., et al. Incidence, characteristics, and outcome of left atrial tachycardias after circumferential antral ablation of atrial fibrillation. (2012) Heart Rhythm 9(10): 1660-1666.

18. Chang, S.L., Tsao, H.M., Lin, Y.J., et al. Differentiating macroreentrant from focal atrial tachycardias occurred after circumferential pulmonary vein isolation. (2011) J Cardiovasc Electrophysiol 22(7): 748-755.

19. Gerstenfeld, E.P., Dixit, S., Bala, R., et al. Surface electrocardiogram characteristics of atrial tachycardias occurring after pulmonary vein isolation. (2007) Heart Rhythm 4(9): 1136-1143.

20. Bochoeyer, A., Yang, Y., Cheng, J., et al. Surface electrocardiographic characteristics of right and left atrial flutter. (2003) Circulation 108(1): 60-66.

21. Marrouche, N.F., Wilber, D., Hindricks, G., et al. Association of atrial tissue fibrosis identified by delayed enhancement MRI and atrial fibrillation catheter ablation: the DECAAF study. (2014) JAMA 311(5): 498-506.

22. Akoum, N., Wilber, D., Hindricks, G., et al. MRI assessment of ablation-induced scarring in atrial fibrillation: analysis from the DECAAF Study. (2015) J Cardiovasc Electrophysiol 26(5): 473-480.

23. McManus, D.D., Tanriverdi, K., Lin, H., et al. Plasma micro RNAs are associated with atrial fibrillation and change after catheter ablation (the miRhythm study). (2015) Heart Rhythm 12(1): 3-10.

24. Sardu, C., Santamaria, M., Paolisso, G., et al. Micro RNA expression changes after atrial fibrillation catheter ablation. (2015) Pharmacogenomics 16(16): 1863-1877.

25. Rizzo, M.R., Sasso, F.C., Marfella, R., et al. Autonomic dysfunction is associated with brief episodes of atrial fibrillation in type 2 diabetes. (2015) J Diabetes Complications 29(1): 88-92.
Ommega Online Publishers

Journal Title: Journal of Heart and Cardiology

Short title : J Heart Cardiol
Journal ISSN: 2378-6914 (online)

Journal E-mail: cardiology@ommegaonline.com

Website: www.ommegaonline.org 